\title{
Effects of Six Sigma methodology on depression and anxiety of patients with end-stage renal disease
}

\author{
Lingling Yang ${ }^{1}$, Haixia Wang ${ }^{1}$, Jingjing Cao ${ }^{1}$, Yin Qian ${ }^{1}$, Yanhong $\mathrm{Gu}^{2}$, Chengmei Chu ${ }^{3}$ \\ ${ }^{1}$ Department of Nephrology, Haian People's Hospital, Nantong, China; ${ }^{2}$ Department of Endocrinology, Haian People's Hospital, Nantong, China; \\ ${ }^{3}$ Department of Gynecology, Haian People's Hospital, Nantong, China \\ Contributions: (I) Conception and design: All authors; (II) Administrative support: C Chu; (III) Provision of study materials or patients: L Yang, H \\ Wang, J Cao, Y Qian, Y Gu; (IV) Collection and assembly of data: L Yang, H Wang, J Cao, Y Qian, Y Gu; (V) Data analysis and interpretation: L \\ Yang; (VI) Manuscript writing: All authors; (VII) Final approval of manuscript: All authors. \\ Correspondence to: Chengmei Chu. Department of Gynecology, Haian People's Hospital, No. 17, Bazhong Road, Nantong 226600 , China.
}

Email: 1228096501@qq.com.

Background: Depression and anxiety are common psychological conditions in end-stage renal disease (ESRD) patients and contribute to poor quality of life and increased mortality. Six Sigma methodology is a novel method of hospital management. The aim of the present study was to determine whether Six Sigma methodology could reduce the incidence of depression and anxiety in ESRD patients.

Methods: ESRD patients undergoing hemodialysis from March 2018 to February 2020 were enrolled in the study. They were divided into the control group or study group according to whether they received conventional nursing care or Six Sigma methodology, respectively. Data on patients' demographic characteristics were retrospectively collected. The conditions of depression and anxiety were assessed by the Self-Rating Depression Scale and the Self-Rating Anxiety Scale, and the severity of depression and anxiety was defined according to the scores of the scales. Univariable and multivariable logistic regression analyses were used to determine the risk factors of depression and anxiety at discharge in ESRD patients and the effects of Six Sigma methodology on them.

Results: A total of 251 patients were retrospectively enrolled in the study, with 112 patients in the control group and 139 patients in the study group. There were no significant differences in the collected demographic characteristics between the two groups. Six Sigma methodology was shown to relieve mild and moderate depression and anxiety at discharge; however, severe depression and anxiety was hardly affected by Six Sigma methodology. The results from multivariable logistic regression analysis identified Six Sigma methodology as an important protective factor of psychological conditions at discharge. Depression and anxiety status at admission and older age are also independent risk factors for anxiety at discharge in ESRD patients. Marital status may also play a role in the incidence of depression.

Conclusions: Six Sigma methodology is significantly useful to reduce the incidence of mild and moderate depression and anxiety at discharge, and should be extended to improve psychological conditions in ESRD patients.

Keywords: Six Sigma methodology; depression; anxiety; end-stage renal disease (ESRD); hemodialysis

Submitted Dec 30, 2020. Accepted for publication Feb 23, 2021.

doi: 10.21037/apm-21-254

View this article at: http://dx.doi.org/10.21037/apm-21-254 


\section{Introduction}

End-stage renal disease (ESRD) is stage 5 of chronic kidney disease and severely worsens the quality of life in these patients (1). Current treatments for ESRD mainly depend on hemodialysis or kidney transplant, and hemodialysis is most commonly used due to insufficient kidney donors. During the long-term treatment of dialysis, some comorbid psychological conditions may occur in ESRD patients psychological and financial pressures, with depression and anxiety being the most common and important conditions (2). Depression is the second comorbid diagnosis after hypertension in ESRD patients undergoing hemodialysis treatment. It has been reported that $23.2 \%$, $86.5 \%$, and $69.1 \%$ of ESRD patients were diagnosed with depression in Saudi Arabia, Iran, and China, respectively (3-5). The incidence of depression was found to be related to poor quality of life and increased mortality in ESRD patients (6). Anxiety is the second comorbid psychological condition in ESRD patients, accounting for $36.9 \%$ of cases in China, and also affects patients' quality of life (5). Depression and anxiety in ESRD patients are reported to be underdiagnosed and undertreated worldwide (7), and the effects of psychological conditions on ESRD patients may be more serious than known. The treatment and management of depression and anxiety should be improved in ESRD patients.

Six Sigma methodology was first proposed for quality control of industry (8), and was then applied in hospital management. There are five procedures in Six Sigma methodology. These are definition, measurement, analysis, improvement, and control, and they aim to eliminate unnecessary steps and process variabilities to reduce errors and improve quality in treatment procedures and nursing care. Six Sigma methodology has been found to reduce the length of hospital stay by minimizing the possible variabilities (9). The rate of cesarean section in pregnancy was reported to decrease from $41.83 \%$ to $32 \%$ by Six Sigma methodology (10). Moreover, Six Sigma methodology has been used in the nursing care of patients with hip fractures (11). The results suggested that Six Sigma methodology contributed to a greater percentage of timely surgical care, lower incidence of complications, and reduced need for resources (11). However, to the best of our knowledge, there is no related study investigating the effects of Six Sigma methodology on comorbid psychological conditions in ESRD patient undergoing hemodialysis.

Six Sigma methodology was gradually applied in hospital management since 1999 in China (12), and it was applied at our hospital within recent several years. In the present study, we retrospectively collected data of ESRD patients receiving different nursing care and investigated whether Six Sigma methodology could reduce the incidence of depression and anxiety in these patients. We present the following article in accordance with the STROBE reporting checklist (available at http://dx.doi.org/10.21037/apm-21-254).

\section{Methods}

\section{Study setting and participants}

The present study was a retrospective study conducted at Haian People's Hospital (Nantong, China) and comprised ESRD patients undergoing hemodialysis from March 2018 to February 2020. All adult patients who underwent hemodialysis for at least 3 months prior to the study were enrolled. The following patients were excluded: those older than 80 years old, suffering from other lifethreating comorbid diseases or psychological diseases, and participating in other clinical trials. Patients were divided into the control and study groups according to the type of nursing care they received. The present study was approved by the Ethics Committee of Haian People's Hospital, but informed consent was not necessary due to it retrospective design. All procedures performed in this study involving human participants were in accordance with the Declaration of Helsinki (as revised in 2013).

\section{Management}

Patients in the control group received routine nursing care, and those in the study group received Six Sigma methodology, which comprised five steps as follows: definition, measurement, analysis, improvement, and control. First, management was defined to reduce the level of psychological conditions in ESRD patients so that patients were willing to cooperate with medical stuff and improve their quality of life. Second, a psychological nursing team was established to focus on the psychological management of ESRD patients. Third, a team meeting was held every week to discuss and analyze the causes of psychological conditions, such as lack of knowledge, economic burden, and decreased self-evaluation. Fourth, improvement measures were discussed and implemented based on the results of the analysis; for example, increasing patients' understanding of the necessity, reliability, and 
safety of hemodialysis, helping patients to use positive selfreward to increase positive behaviors, guiding patients to learn relaxation techniques to promote feelings of comfort, and encouraging patients to identify and reduce their negative thinking. Finally, the function of the control step was the implementation of health education, strengthening the importance of psychological nursing.

\section{Data collection}

Demographic and clinical data were collected, including age, sex, body mass index (BMI), education status (none, primary, secondary, tertiary), marital status (married, single, divorced, widowed, separated), employment status, and duration of renal failure, to investigate their relationship with the incidence of depression and anxiety.

\section{Outcome assessment}

The outcomes in the present study were the incidences of depression and anxiety in ESRD patients at admission and discharge. The incidence and level of depression and anxiety were assessed using the Self-Rating Depression Scale (SDS) and the Self-Rating Anxiety Scale (SAS). There were four grades in both scales; 1 point indicated no or seldom, 2 points indicated sometimes, 3 points indicated most of the time, and 4 points indicated almost all of the time. The cut-off value of the SDS was 53 points, 53-62 points for mild depression, 63-72 points for moderate depression, and $>72$ points for severe depression. The cut-off value of the SAS was 50 points, 50-59 points for mild anxiety, 60-69 points for moderate anxiety, and $>70$ points for severe anxiety.

\section{Statistical analysis}

Continuous variables in the present study were converted into categorical variables according to defined cutoff. Categorical variables were reported as number and percentage, and the comparisons between categorical variables were based on $\chi^{2}$-test. To determine the risk factors of depression and anxiety at discharge in ESRD patients and the effects of Six Sigma methodology on them, univariable and multivariable logistic regression analyses were performed. $\mathrm{P}<0.05$ indicated statistically significant differences, and all statistical analyses was performed using SPSS 20.0 (IBM, Armonk, NY, USA).

\section{Results}

We collected data from 251 ESRD patients undergoing hemodialysis during a period of 2 years. These patients were then divided into two groups, with 112 patients in the control group receiving conventional nursing care and 139 patients study group receiving Six Sigma methodology. The characteristics of the enrolled patients were listed in Table 1 . The majority of patients were relatively older patients; patients $>40$ years old accounted for $64.5 \%$ of the total number of patients. Around $68.1 \%$ patients were males, and the BMI of nearly half of patients was $<20$. Only $18.7 \%$ patients had a tertiary education and $80.1 \%$ patients were married and lived with their partner. More than half of the enrolled patient had undergone hemodialysis for more than 1 year and $<3$ years, and $34.2 \%$ patients had undergone hemodialysis for more than 3 years. We also recorded the patients' psychological conditions and found that that $47.8 \%$ and $36.3 \%$ of patients were diagnosed as having depression and anxiety at admission, respectively. There were no significant differences in the collected characteristics between the study group and the control group.

The incidences of depression and anxiety at discharge were recorded as outcome variables, as shown in Table 2. It was found that the incidence of depression was $50.9 \%$ in the control group and $39.6 \%$ in the study group, indicating a significant difference between them $(\mathrm{P}=0.018)$. Similarly, the incidence of anxiety was $27.6 \%$ in the study group, which was much lower than the $44.6 \%$ reported in the control group $(\mathrm{P}=0.002)$. The change in different levels of depression in both groups is shown in Figure 1. It was noted that some patients with mild and moderate depression could be relieved after Six Sigma methodology, and routine nursing care was found to not have a significant impact on patients' emotion. The change in different levels of anxiety is shown in Figure 2. Six Sigma methodology was effective in relieving mild and moderate anxiety according to the results. However, Six Sigma methodology was not significantly beneficial to the relief of depression and anxiety in the present study.

Finally, we determined the risk factors of depression using logistic regression analysis, as shown in Table 3. The results from the multivariable regression analysis indicated that depression and anxiety status at admission was related to the incidence of depression at discharge. Older age and living alone were also important risk factors of depression at discharge. Most importantly, Six Sigma methodology was 
Table 1 Characteristics of study population at admission

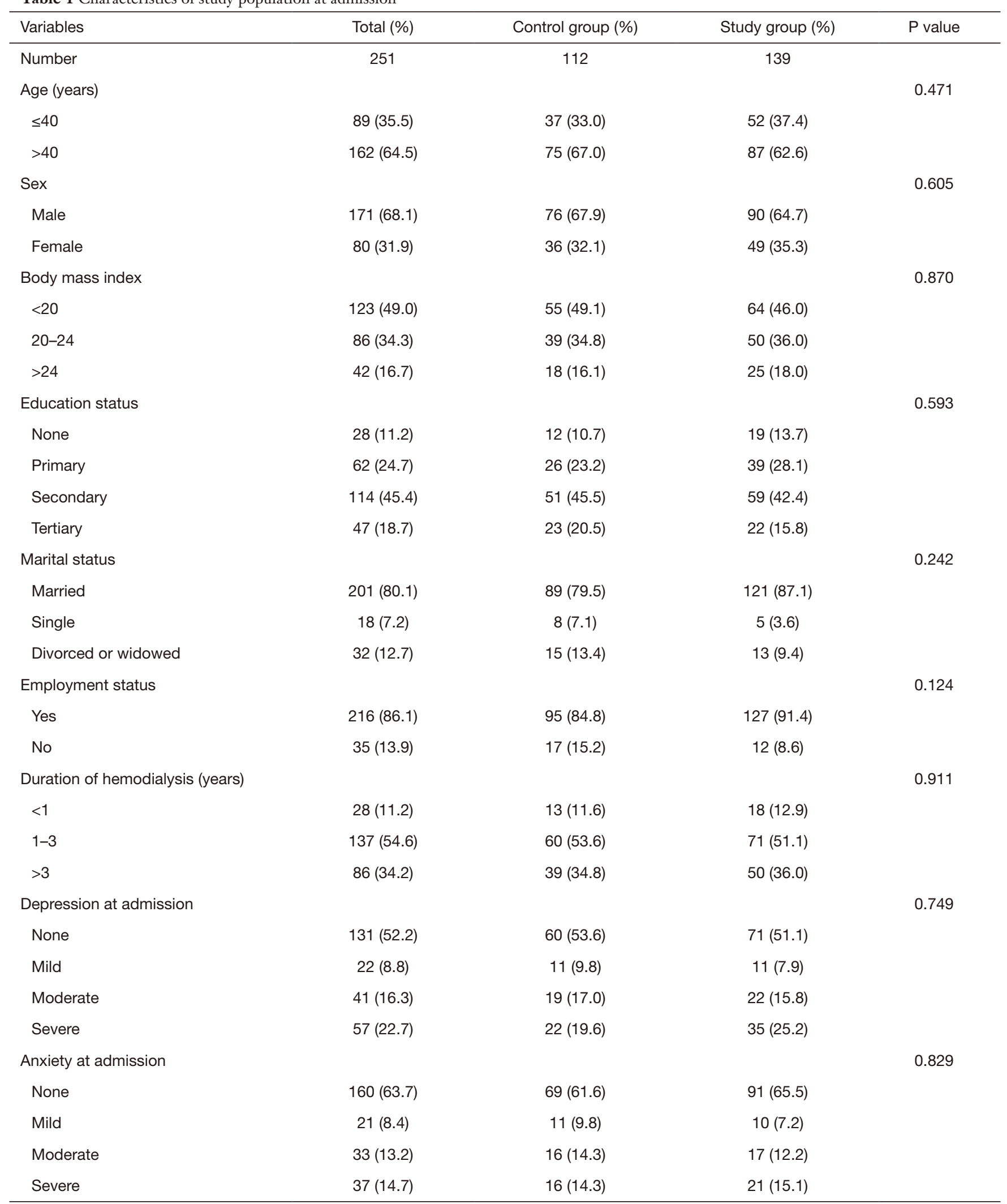


Table 2 Incidence of depression and anxiety of study population at discharge

\begin{tabular}{lccc}
\hline Variables & Total $(\%)$ & Control group (\%) & Study group (\%) \\
\hline Number & 251 & 112 & 139 \\
Depression at discharge & & & 0.018 \\
None & $139(55.4)$ & $55(49.1)$ & $54(60.4)$ \\
Mild & $21(8.4)$ & $14(12.5)$ & $5(5.0)$ \\
Moderate & $37(14.7)$ & $20(17.9)$ & $16(12.2)$ \\
Severe & $54(21.5)$ & $23(20.5)$ & $34(22.3)$ \\
Anxiety at discharge & & $62(55.4)$ & $102(73.4)$ \\
None & $164(65.3)$ & $15(13.4)$ & $4(5.0)$ \\
Mild & $22(8.8)$ & $17(15.2)$ & $11(9.4)$ \\
Moderate & $30(12.0)$ & $18(16.1)$ & $22(12.2)$ \\
Severe & $35(13.9)$ & & 0.02 \\
\hline
\end{tabular}

Italic values indicate significant difference.
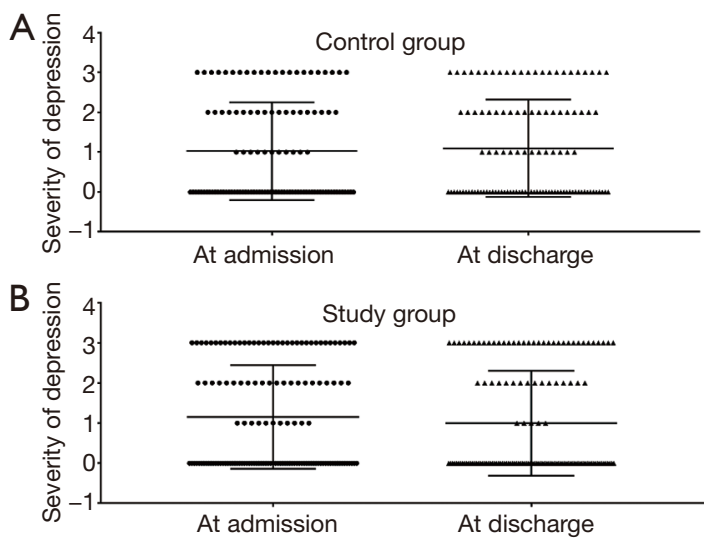

Figure 1 Severity of depression at admission and discharge in two group. (A) Control group; (B) study group. 0, none; 1, mild depression; 2 , moderate depression; 3 , severe depression.

found to be a protective factor of depression at discharge in ESRD patients $(\mathrm{P}=0.032)$. Logistic regression analysis was then used to determine the risk factors of anxiety, as shown in Table 4. Depression and anxiety status at admission and older age were also independent risk factors for anxiety at discharge in ESRD patients. Living alone showed no relationship with anxiety at discharge after adjusting with other confounding factors in the multivariable regression analysis. Six Sigma methodology was found to reduce the incidence of anxiety at discharge $(\mathrm{P}=0.003)$.

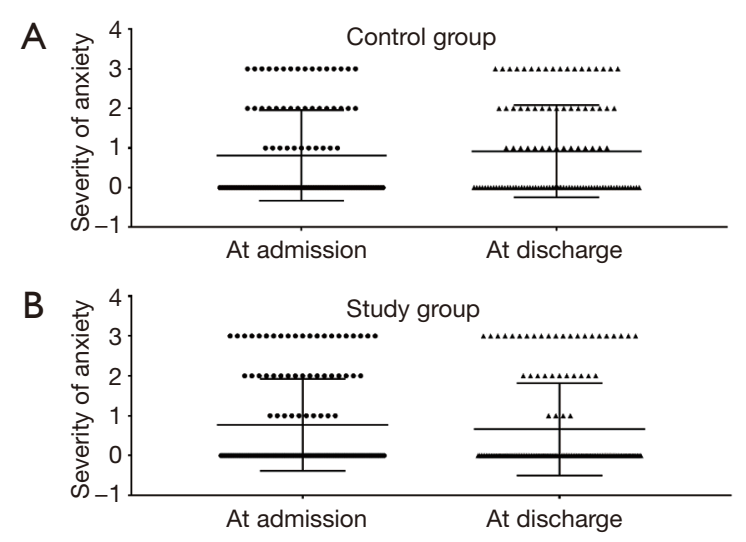

Figure 2 Severity of anxiety at admission and discharge in two groups. (A) Control group; (B) study group. 0, none; 1, mild anxiety; 2 , moderate anxiety; 3 , severe anxiety.

\section{Discussion}

In the present study, we summarized the data of ESRD patients undergoing hemodialysis at a single center within a period of 2 years. The incidence of depression at discharge was $44.6 \%$ and that of anxiety at discharge was $34.7 \%$. These incidences were similar to those reported in previously published studies $(5,13)$, indicating that psychological conditions are common in ESRD patients. Previous studies have indicated that depression and anxiety worsen quality of life in ESRD patients $(14,15)$. Efforts 
Table 3 Univariable logistic regression analysis of risk factors of depression in end-stage renal disease patients

\begin{tabular}{|c|c|c|c|c|}
\hline Variables & \multicolumn{2}{|c|}{ Univariable analysis } & \multicolumn{2}{|c|}{ Multivariable analysis } \\
\hline Six Sigma vs. conventional care & $0.484(0.292-0.804)$ & 0.005 & $0.553(0.321-0.951)$ & 0.032 \\
\hline Age ( $\leq 40$ vs. $>40$ years) & $0.423(0.254-0.705)$ & 0.001 & $0.400(0.232-0.690)$ & 0.001 \\
\hline Female vs. male & $1.023(0.600-1.744)$ & 0.934 & - & - \\
\hline Tertiary education vs. other & $0.810(0.425-1.543)$ & 0.521 & - & - \\
\hline Married vs. other & $0.558(0.331-0.941)$ & 0.029 & $0.582(0.376-0.982)$ & 0.045 \\
\hline Employed vs. unemployed & $1.053(0.514-2.156)$ & 0.889 & - & - \\
\hline$>3$ years of renal failure $v s . \leq 3$ years & $1.250(0.759-2.059)$ & 0.380 & - & - \\
\hline
\end{tabular}

Italic values indicate significant difference. $\mathrm{Cl}$, confidence interval; OR, odds ratio.

Table 4 Univariable logistic regression analysis of risk factors of anxiety in end-stage renal disease patients

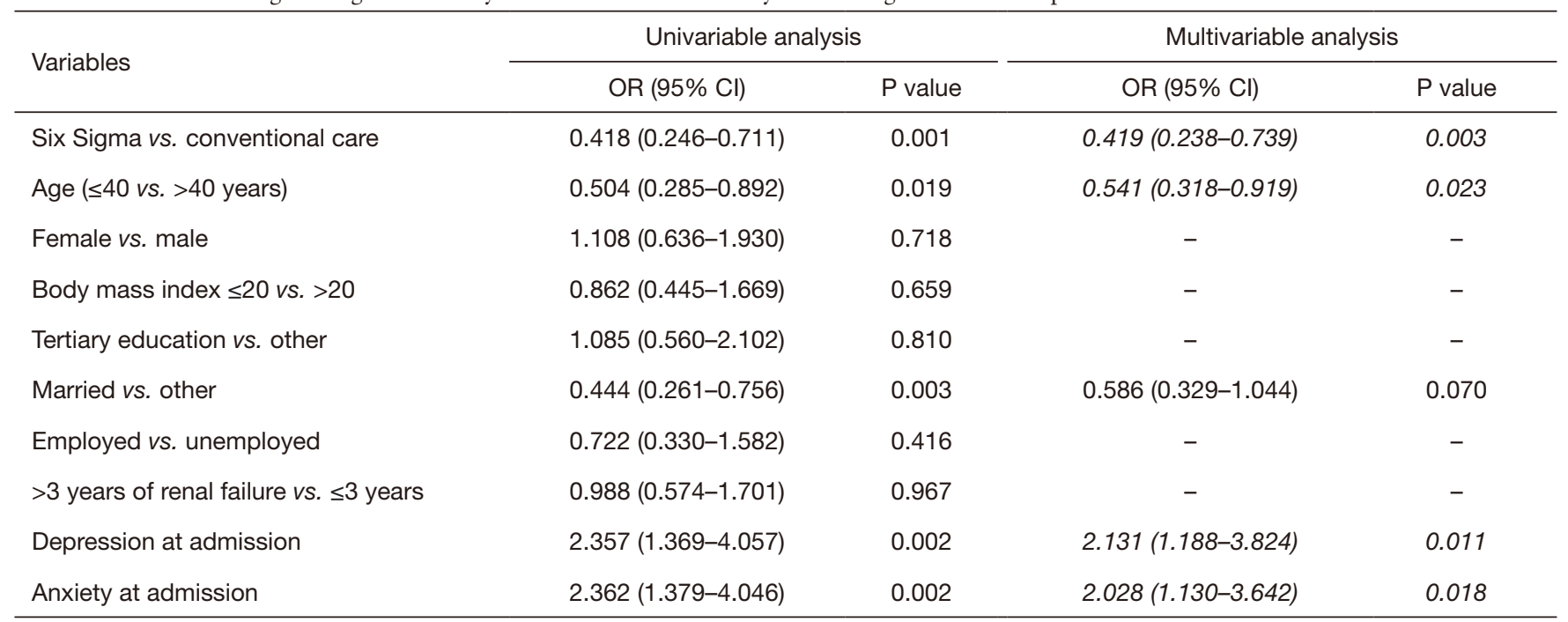

Italic values indicate significant difference. $\mathrm{Cl}$, confidence interval; OR, odds ratio.

have been made to relieve psychological conditions using psychotropic medications or non-pharmacological therapy $(16,17)$. According to a previous study, only a small group of ESRD patients with depression received either psychotropic medications or non-pharmacological therapy (18). It is still unclear whether psychotropic medications are tolerable and efficacious in ESRD patients. The effectiveness of nonpharmacological therapy, which mainly refers to cognitivebehavioral therapy, was confirmed in several studies with small sample sizes $(19,20)$.
Six Sigma methodology was applied in the present study and showed significantly positive effects on the psychological conditions of ESRD patients. To the best of our knowledge, the present study is the first to investigate the effects of Six Sigma methodology on the incidence of depression and anxiety in ESRD patients. Previous studies have confirmed that Six Sigma methodology could improve the timeliness of care and the overall quality of nursing care in several departments $(21,22)$. We then modified conventional Six Sigma methodology and added 
some cognitive-behavioral therapy into it to focus on the improvement of psychological conditions in ESRD patients. Our results further confirmed the effectiveness of cognitivebehavioral therapy in ESRD patients, with modified Six Sigma methodology being identified as a protective factor of depression and anxiety at discharge. In contrast, conventional nursing care without specific psychological treatment would worsen psychological conditions to some degree.

After further analysis, it was found that Six Sigma methodology mainly relieved mild depression and anxiety in the present study. Severe depression and anxiety were hard to eradicate completely or could only be slightly relieved, which was a shortcoming of Six Sigma methodology. The addition of psychotropic medications may be helpful. However, treatment using psychotropic medications is difficult to implement in practice. The main reason is that patients are reluctant to accept psychotropic medications. A lack of studies investigating the effects of psychotropic medications on ESRD patients at present also hinders their application for the treatment of depression and anxiety. Such studies are necessary for the treatment of ESRD patients with depression and anxiety.

Other risk factors of depression and anxiety were also identified in the present study. Age was a significant predictor of anxiety and depression, which was similar to the results of a previous study (3). Married patients were more likely to be depressed or anxious according to our study. However, previous studies have different opinions on the impacts of marital status on psychological condition $(7,13)$. We believe that marital status and living with family is more conducive to the psychological condition of patients, as they provide patients with support and reduce their psychological stress.

The present study has some limitations. First, most patients underwent hemodialysis for a relatively short period ( $<3$ years, $65.8 \%$ ). Therefore, it was unclear whether these results are applicable to patients who have undergone hemodialysis for longer periods of time. Second, we did not record the prognosis and quality of life due to the short follow-up of less than 3 years. Third, depression and anxiety were diagnosed by the SAS and SDS scores instead of a psychiatrist. Some novel scales to assess depression and anxiety have been proposed in recent years, which may be superior to the SDS and SAS $(2,23,24)$. Further studies are needed to verify their effectiveness in ESRD patients.

\section{Conclusions}

In total, 251 ESRD patients undergoing hemodialysis were enrolled in the present study and the incidence of depression and anxiety at discharge was $44.6 \%$ and $34.7 \%$, respectively. Six Sigma methodology was significantly useful to reduce the incidence of depression and anxiety at discharge, especially mild and moderate depression and anxiety. The findings of the present study indicated that Six Sigma methodology should be extended to improve psychological conditions in ESRD patients.

\section{Acknowledgments}

Funding: This work was supported by the Social Livelihood Science and Technology Foundation of Nantong (MSZ18190).

\section{Footnote}

Reporting Checklist: The authors have completed the STROBE reporting checklist. Available at http://dx.doi. org/10.21037/apm-21-254

Data Sharing Statement: Available at http://dx.doi. org/10.21037/apm-21-254

Conflicts of Interest: All authors have completed the ICMJE uniform disclosure form (available at http://dx.doi. org/10.21037/apm-21-254). The authors have no conflicts of interest to declare.

Ethical Statement: The authors are accountable for all aspects of the work in ensuring that questions related to the accuracy or integrity of any part of the work are appropriately investigated and resolved. The present study was approved by the Ethics Committee of Haian People's Hospital, but informed consent was not necessary due to it retrospective design. All procedures performed in this study involving human participants were in accordance with the Declaration of Helsinki (as revised in 2013).

Open Access Statement: This is an Open Access article distributed in accordance with the Creative Commons Attribution-NonCommercial-NoDerivs 4.0 International License (CC BY-NC-ND 4.0), which permits the noncommercial replication and distribution of the article with 
the strict proviso that no changes or edits are made and the original work is properly cited (including links to both the formal publication through the relevant DOI and the license). See: https://creativecommons.org/licenses/by-nc-nd/4.0/.

\section{References}

1. Gagnier SA, Pieper BA. An Integrative Review of Depression in Patients Receiving Hemodialysis for Endstage Renal Disease and the Relevance to Patients With Wounds. Wound Manag Prev 2019;65:28-34.

2. Wang J, Zhou T, Liu J, et al. Application of (1)H-MRS in end-stage renal disease with depression. BMC Nephrol 2020;21:225.

3. Turkistani I, Nuqali A, Badawi M, et al. The prevalence of anxiety and depression among end-stage renal disease patients on hemodialysis in Saudi Arabia. Ren Fail 2014;36:1510-5.

4. Norozi Firoz M, Shafipour V, Jafari H, et al. Relationship of Hemodialysis Shift With Sleep Quality and Depression in Hemodialysis Patients. Clin Nurs Res 2019;28:356-73.

5. Hou Y, Li X, Yang L, et al. Factors associated with depression and anxiety in patients with end-stage renal disease receiving maintenance hemodialysis. Int Urol Nephrol 2014;46:1645-9.

6. Yoong RK, Mooppil N, Khoo EY, et al. Prevalence and determinants of anxiety and depression in end stage renal disease (ESRD). A comparison between ESRD patients with and without coexisting diabetes mellitus. J Psychosom Res 2017;94:68-72.

7. Semaan V, Noureddine S, Farhood L. Prevalence of depression and anxiety in end-stage renal disease: A survey of patients undergoing hemodialysis. Appl Nurs Res 2018;43:80-5.

8. Oosterhuis WP, Severens MJ. Performance specifications and six sigma theory: Clinical chemistry and industry compared. Clin Biochem 2018;57:12-7.

9. Prajapati D, Suman G. Six sigma approach for neonatal jaundice patients in an Indian rural hospital - a case study. Int J Health Care Qual Assur 2019. [Epub Ahead of Print]. doi: 10.1108/IJHCQA-07-2019-0135.

10. Chai ZY, Hu HM, Ren XL, et al. Applying Lean Six Sigma methodology to reduce cesarean section rate. J Eval Clin Pract 2017;23:562-6.

11. Sayeed Z, Anoushiravani A, El-Othmani M, et al. Implementation of a Hip Fracture Care Pathway Using Lean Six Sigma Methodology in a Level I Trauma Center. J Am Acad Orthop Surg 2018;26:881-93.
12. Zhang C, Zhao H, Wang J, et al. The Application of Six Sigma Techniques in the Evaluation of Enzyme Measurement Procedures in China. Clin Lab 2015;61:461-5.

13. Al-Shammari N, Al-Modahka A, Al-Ansari E, et al. Prevalence of depression, anxiety, and their associations among end-stage renal disease patients on maintenance hemodialysis: a multi-center population-based study. Psychol Health Med 2020. [Epub ahead of Print]. doi: 10.1080/13548506.2020.1852476.

14. Barros A, Costa BE, Mottin CC, et al. Depression, quality of life, and body composition in patients with end-stage renal disease: a cohort study. Braz J Psychiatry 2016;38:301-6.

15. Goh ZS, Griva K. Anxiety and depression in patients with end-stage renal disease: impact and management challenges - a narrative review. Int J Nephrol Renovasc Dis 2018;11:93-102.

16. Hedayati SS, Yalamanchili V, Finkelstein FO. A practical approach to the treatment of depression in patients with chronic kidney disease and end-stage renal disease. Kidney Int 2012;81:247-55.

17. Hedayati SS, Daniel DM, Cohen S, et al. Rationale and design of A Trial of Sertraline vs. Cognitive Behavioral Therapy for End-stage Renal Disease Patients with Depression (ASCEND). Contemp Clin Trials 2016;47:1-11.

18. Weisbord SD, Mor MK, Green JA, et al. Comparison of symptom management strategies for pain, erectile dysfunction, and depression in patients receiving chronic hemodialysis: a cluster randomized effectiveness trial. Clin J Am Soc Nephrol 2013;8:90-9.

19. Cukor D, Ver Halen N, Asher DR, et al. Psychosocial intervention improves depression, quality of life, and fluid adherence in hemodialysis. J Am Soc Nephrol 2014;25:196-206.

20. Duarte PS, Miyazaki MC, Blay SL, et al. Cognitivebehavioral group therapy is an effective treatment for major depression in hemodialysis patients. Kidney Int 2009;76:414-21.

21. Flanary JT, Rocco NR, Dougherty T, et al. Use of Lean Six Sigma to Improve Access to Care in a Surgical Subspecialty Clinic. Mil Med 2020;185:e887-e893.

22. Davies C, Lyons C, Whyte R. Optimizing nursing time in a day care unit: Quality improvement using Lean Six Sigma methodology. Int J Qual Health Care 2019;31:22-8.

23. Neitzer A, Sun S, Doss S, et al. Beck Depression 
Inventory-Fast Screen (BDI-FS): an efficient tool for depression screening in patients with end-stage renal disease. Hemodial Int 2012;16:207-13.

24. AlAwwa I, Ibrahim S, Obeid A, et al. Comparison of pre- and post-hemodialysis PHQ-9 depression scores in

Cite this article as: Yang L, Wang H, Cao J, Qian Y, Gu Y, Chu C. Effects of Six Sigma methodology on depression and anxiety of patients with end-stage renal disease. Ann Palliat Med 2021;10(4):4375-4383. doi: 10.21037/apm-21-254 patients with end-stage renal disease: A cross-sectional study. Int J Psychiatry Med 2020. [Epub ahead of print]. doi: 10.1177/0091217420973489.

(English Language Editor: R. Scott) 